\title{
A Case of Wegener's Granulomatosis Treated with Mycophenolate Mofetil
}

\author{
Song Lin Lin Shan Qiu Mingcai \\ Kidney Department, Tianjin Medical University, General Hospital, Tianjin, PROC
}

Dear Sir,

A 56-year-old male patient, 3 years before the admission, had ocular pain, photophobia and dacryorrhea without inducement, was diagnosed as scleritis and treated with prednisone $30 \mathrm{mg} / \mathrm{day}$ for 2 months. The therapy had to be stopped because of secondary diabetes mellitus. Four months after the onset, the patient intermittently nosebleeding and hoarseness. Chest X-ray films and CT scans revealed multiple nodules in both lungs which implied inflammation by MRI, CANCA positive and proteinuria, the patient received nodules biopsy and was diagnosed as Wegener's granulomatosis (WG). Given the treatment with prednisone $40 \mathrm{mg} /$ day and total dose of $15 \mathrm{~g}$ of cyclophamide for 4 months until remission (C-ANCA negative and lung nodules disapeared), the patient received maintenance therapy with low dose prednisone $15 \mathrm{mg} /$ q.o.d. for 18 months. The patient was on hemodialysis because renal function became worse and eventually developed to uremia 8 months ago. After 3 months of hemodialysis, a series of symptoms appeared such as low fever, headache, nausea and vomiting, cough, extremities numbness. Chest X-ray films and CT scans revealed one nodule again and C-ANCA turned to be positive, head MRI showed abnormal signal associated with vasculities in left basal ganglia and right maxillary sinusitis. Diagnosed as relapse of WG, the patient received a therapy of low dose prednisone $30 \mathrm{mg} / \mathrm{q}$.o.d. and mycophenolate mofetil (MMF) $1.5 \mathrm{~g} /$ day. After the treatment for 3 months, the clinical symptoms described above disappeared, C-ANCA became negative and lung nodule disappeared again. The patient received successful remission and renal function returned back to be better without drug-related adverse effects.

WG comprises the triad of systemic vasculitis associated with granulomatous inflammation in the upper and lower respiratory tracts and glomerulonephritis. The pathogenesis of WG is associated with abnormalities of both humoral and cell-mediated immunity, including immune complex-mediated mechanism, autoantibody-mediated mechanism, ANCA-induced neutrophil degranulation, Tlymphocyte-mediated mechanism. WG typically is a multisystem disease with many organs involved, including nose, eye, ear, respiratory tract, kidney, nervous system, gastrointestinal tract, heart and skin. In the present case, during the course of two and half years between onset to relapse, multisystems were involved gradually with severe renal lesion, indicating that early diagnosis of WG relies on biopsy and ANCA assays, and MRI is better than CT for the diagnosis of immunovasculitis. The treatment of WG is corticosteroids combined with cytotoxic immunosuppressive drug (cyclophosphamide, azathioprine, etc.). The prognosis of WG is expected to be bad and most patients usually die from renal failure.

Mycophenolate mofetil is a potential new immunosuppressive drug, which is the 2morpholinoethyl ester of mycophenolic acid (MPA), MPA is a non-competitive and reversible inhibitor of inosine monophosphate dehydrogenase. This enzyme is required by lymphocytes for the synthesis of guanosine nucleotides, DNA production and cell proliferation. MMF is rapidly absorbed after oral administration and undergoes complete hydrolysis to MPA. MMF can suppress the proliferation of $\mathrm{T}$ and $\mathrm{B}$ lymphocytes, bloch the glycosylation of adhesion molecules and reduce the function of lymphocyte adhesion and infiltration to the vascular endothelium, inhibit production of antibodies from lymphocytes and spleen cells.

MMF was used not only in transplant field, but also in autoimmune diseases recently. Due to relapse of WG after 2 years of maintenance therapy, the patient received low-dose prednisone and MMF for 3 months, then received successful remission without gastrointestinal tract reaction, bone marrow depression and liver lesion. It could be concluded that MMF in combination with lowdose corticosteroids could be well tolerated and effective for WG. Long-term treatment with MMF is encouraging because of its low toxicity. The mechanism was most likely that MMF suppressed ANCA production and lymphocytes proliferation and reduced the incidence of vasculitis mediated by immunocytes and inflammatory materials. The treatment of WG with MMF remains to be further studied and more clinical trials.

\section{References}

1 Jayne D: Non-transplant uses of mycophenolate mofetil. Curr Opin Nephrol Hypertens 1999;8:563-567.

2 Nowack R, Gobel U, Klooker P, et al: Mycophenolate mofetil for maintenance therapy of Wegener's granulomatosis and microscopic polyangiitis. J Am Soc Nephrol 1999;10:19651971.

\section{KARGER}

Fax +41613061234 E-Mail karger@karger.ch www.karger.com (c) 2002 S. Karger AG, Basel 0028-2766/02/0924-0959\$18.50/0

Accessible online at: www.karger.com/journals/nef
Song Lin

Kidney Department, General Hospital of Tianjin Medical University

154 Anshan Road, Heping District

300052 Tianjin (People's Republic of China)

Tel. +86 22278161102598 , Fax +8622 26720668, E-Mail Songlin@public.tjuc.com.cn 